\title{
The spread of infectious diseases in spatially structured populations: an invasory pair approximation
}

\author{
Chris T. Bauch
}

revised for Mathematical Biosciences

27 May 2005

first submitted

28 April 2004 
Contact Details:

Department of Mathematics and Statistics

University of Guelph

50 Stone Road East

Guelph, Ontario N1G 2W1

Canada

Email: cbauch@uoguelph.ca

Phone: 519-824-4120 ext. 53079

Fax: 519-837-0221 


\begin{abstract}
The invasion of new species and the spread of emergent infectious diseases in spatially structured populations has stimulated the study of explicit spatial models such as cellular automata, network models and lattice models. However, the analytic intractability of these models calls for the development of tractable mathematical approximations that can capture the dynamics of discrete, spatially-structured populations. Here we explore moment closure approximations for the invasion of an SIS epidemic on a regular lattice. We use moment closure methods to derive an expression for the basic reproductive number, $\mathcal{R}_{0}$, in a lattice population. On lattices, $\mathcal{R}_{0}$ should be bounded above by the number of neighbors per individual. However, we show that conventional pair approximations actually predict unbounded growth in $\mathcal{R}_{0}$ with increasing transmission rates. To correct this problem, we propose an 'invasory' pair approximation which yields a relatively simple expression for $\mathcal{R}_{0}$ that remains bounded above, and also predicts $\mathcal{R}_{0}$ values from lattice model simulations more accurately than conventional pair and triple approximations. The invasory pair approximation is applicable to any spatial model, since it takes into account characteristics of invasions that are common to all spatially structured populations.
\end{abstract}

Keywords: contact process, SIS model, basic reproductive number, moment closure approximations, pair approximations, lattice models 


\section{Introduction}

Invasive species and emergent infectious diseases continue to threaten many populations in which spatial structure is important. This has stimulated the development of numerous explicit spatial models which, although less analytically tractable than their traditional non-spatial counterparts, are often valuable for modelling spatial dynamics of invasion $[1,2]$. Because of the importance of spatial structure, modellers continue to study how to incorporate spatial structure in population models without losing too much analytic tractability.

Predictions of spatial models often differ significantly from those of their nonspatial counterparts $[1,3]$. In spatially structured populations, it is easier for coexistence, diversity and altruism to develop [4,5], evolutionary velocities are slower [6], and epidemics have more realistic time series and critical community sizes [7]. In a mean-field (non-spatial) approximation, the population is assumed to be well-mixed, such that each individual is equally likely to contact any other individual in the population. Hence, the success or failure of an invasion depends only upon non-spatial parameters such as transmission rates or recovery rates. By comparison, in a spatially structured population, spatial structure also plays a role in determining the success or failure of the invasion $[3,8,9]$.

As computational power continues to increase, spatially explicit, individual-based models such as cellular automata and lattice models have been increasingly applied to the study of spatial dynamics $[2,10,11]$. Individual-based models are easy to im-

plement, and can incorporate any level of detail. However they are also analytically intractable, their implementation can vary across studies in subtle (and often unstated) but important ways, and their output may be difficult to interpret reliably. Accordingly there is a need for mathematical approximations to spatially explicit models to help researchers better understand spatial dynamics, to illustrate general principles and to identify which factors are most crucial in spatially structured populations. One such approximation technique that has attracted significant interest in 
the past decade is moment closure approximation [3, 4, 8, 9, 12-24]. Moment closure approximations are increasingly recognized as a valuable tool for analyzing spatial dynamics in populations where stochastic, discrete (individual-level) spatial interactions are important.

To derive a moment closure approximation, one first writes down the equations of motion for the number of neighboring pairs of individuals of a given type on a lattice/network (or pairs separated by a certain distance, if individuals are distributed across continuous space). Typically these equations have terms involving triples. When the equations of motion for the triples are derived, terms involving quadruples appear, etc. The result is a potentially infinite hierarchy of equations of motion. To obtain a manageable set of equations one must truncate the hierarchy at some suitable level, a process known as moment closure. The closure makes an assumption about the statistical distributions that govern local spatial structure, and particularly the distribution which relates higher-order quantities to the quantities at the level of the truncation. When the closure is made at the level of pairs (such that third-order quantities are approximated in terms of second-order quantities), the result is usually called a pair approximation.

One of the simplest examples of a spatial individual-based model is the SIS epidemic on a lattice, which is formally equivalent to the contact process [25]. Individuals are distributed across the nodes of a regular lattice (for example, square or hexagonal). Each lattice node is occupied by an individual who is either susceptible to the disease, or infected (and infectious). Each individual can interact only with his or her $Q$ nearest neighbors, where $Q$ is the number of lattice edges emanating from each node ( $Q=6$ for a hexagonal lattice, for example). Hence each individual is a member of $Q$ pairs. An infected individual infects a neighboring susceptible individual at rate $\lambda$, and infected individuals recover to the susceptible state at rate $\nu$. This SIS (susceptible $\rightarrow$ infected $\rightarrow$ susceptible) scheme is applicable to diseases where there is a lack of acquired immunity, e.g., many bacterial diseases in higher vertebrates, 
as well as many invertebrate and plant diseases. We assume a large population on a regular, two-dimensional lattice with periodic (toroidal) boundary conditions. If $N$ is the population size, we require $Q \ll N$. As $Q \rightarrow N-1$, the population becomes increasingly homogeneously mixed, and spatial structure becomes irrelevant to epidemiological dynamics.

In this paper we use pair approximations to study invasion of an SIS epidemic on a lattice. Transient solutions (such as trajectories of invasion) of pair approximations have generally received less attention than their equilibrium solutions. However, invasion is of major ecological and epidemiological importance. Here we use moment closure techniques to derive expressions for the basic reproductive number $\mathcal{R}_{0}$, defined as the average number of new infections produced by a typical infected individual in a wholly susceptible population [26-28]. For $\mathcal{R}_{0}>1$ an epidemic may occur, whereas for $\mathcal{R}_{0} \leq 1$, the disease fades out stochastically before infecting a significant fraction of the population. $\mathcal{R}_{0}$ provides a criterion for whether an invasion can succeed, and consequently it is of major importance in quantifying the feasibility of epidemic control. (In the ecological analogue, a similar measure $\mathcal{R}$ (the fecundity of a species) determines whether or not a new species can successfully invade a territory.)

If the above definition of $\mathcal{R}_{0}$ is applied to a lattice population, then $\mathcal{R}_{0}$ should be bounded above by $Q$, since an infected individual can only infect his or her $Q$ neighbours (in fact, for the SIS model, $\mathcal{R}_{0}$ should be bounded above by $Q-1$, as we will discuss in Section 4). Previous work has shown that is possible to compute an expression for $\mathcal{R}_{0}$ in a lattice population using moment closure techniques that can take spatial structure partially into account $[3,9]$. We show here that when this technique is applied using the standard ordinary pair approximation (OPA) [4,9], it predicts unbounded growth in $\mathcal{R}_{0}$ as the transmission rate $\lambda \rightarrow \infty$. This is also true for a previously proposed pair approximation designed to improve upon the OPA by taking spatial clumping into account $[18,29]$. Here, we propose an invasory pair approximation (IVPA) that circumvents these problems by taking into account the 
spatial structure of invading clusters in the early stages of invasion. When $\mathcal{R}_{0}$ is derived using the IVPA instead, we obtain a relatively simple, closed-form expression for $\mathcal{R}_{0}$ that is bounded above by $Q+1 / 2$ as $\lambda \rightarrow \infty$, and which also agrees better with $\mathcal{R}_{0}$ values computed directly from lattice model simulations. A triple approximation is also derived, and predicts an $\mathcal{R}_{0}$ bounded above by $Q+3 / 2$ as $\lambda \rightarrow \infty$, however the accuracy is worse than the IVPA and the method does not yield a simple, closed-form expression for $\mathcal{R}_{0}$.

In Section 2 we derive a mean-field approximation and in Section 3 we derive the three pair approximations and the triple approximation to the SIS epidemic on a lattice. In Section 4 we derive an expression for $\mathcal{R}_{0}$ using the various moment closure approximations, and we compare the approximations to results from stochastic individual-based simulations of the lattice model. We conclude the paper with a discussion in Section 5.

\section{Mean-field approximation}

The first step in deriving the mean-field approximation is to write down the equation of motion for $[I]$, the number of infected individuals. We assume that the total population size is constant, hence $[I]$ is related to the number $[S]$ of susceptible individuals by $[I]+[S]=N$, where $N$ is the population size. Infected individuals transmit the infection to susceptible neighbors at rate $\lambda$, so the equation of motion for $[I]$ includes the number $[S I]$ of susceptible-infected pairs:

$$
\frac{d}{d t}[I]=-\nu[I]+\lambda[S I]
$$

Under the mean-field approximation, spatial structure is ignored altogether. Susceptibles and infecteds are assumed to mix homogeneously, such that an infection can be passed from an infected individual to potentially any susceptible individual. In the lattice model formalism this is equivalent to setting $Q=N-1$. Hence $[S I]=[S][I]$, 
and the equation of motion for $[I]$ under a mean-field approximation becomes

$$
\frac{d}{d}[I]=-\nu[I]+\lambda[S][I]=-\nu[I]+\lambda(N-[I])[I]
$$

The mean-field approximation is the moment closure of lowest order, since we close the equations of motion by replacing the second-order quantity $[S I]$ with two first-order quantities $[S]$ and $[I]$. It will often be convenient to express the rate of disease transmission in terms of the parameter

$$
\beta \equiv \lambda Q
$$

where $\beta$ can be thought of as the overall transmission rate, taking the neighborhood size into account. As $Q$ increases, $\lambda$ can be decreased accordingly, in situations where it is desirable to keep the overall disease transmissibility of an individual constant.

\section{Moment closure approximations}

Since $Q \ll N$ for spatial populations, it is clear that the homogeneous mixing approximation may lead to inaccuracies. An alternative to setting $[S I]=[S][I]$ is to retain $[S I]$ as a state variable, and to write down its equation of motion. This constitutes the first step in building spatial structure implicitly into a system of differential equations for approximating an SIS epidemic on a lattice.

We will derive the equation of motion for $[S I]$ as an illustrative example of moment closure approximations (details are in Appendix A). The derivation starts by listing all events that can change the number of susceptible-infected pairs, and then summing, over all relevant pairs at which these events occur, their rate of occurrence multiplied by the resulting change in $[S I]$. One thus obtains:

$$
\frac{d}{d t}[S I]=-\nu[S I]+\nu[I I]-\lambda[S I] Q(I \mid S I)+\lambda[S S] Q(I \mid S S)
$$

where $[I I]$ and $[S S]$ denote twice the number of infected-infected and susceptiblesusceptible pairs respectively (double counting simplifies the equations of motion), 
and where $Q(I \mid S I)$ (resp. $Q(I \mid S S)$ ) is the average number of infected neighbors of a susceptible, given that the susceptible already has at least one infected (resp. susceptible) neighbor. The equations of motion for $[I I]$ and $[S S]$ are

$$
\begin{aligned}
\frac{d}{d t}[I I] & =-2 \nu[I I]+2 \lambda[S I] Q(I \mid S I), \\
\frac{d}{d t}[S S] & =2 \nu[S I]-2 \lambda[S S] Q(I \mid S S) .
\end{aligned}
$$

In order to close Eqs. (4)-(6), we must express the third-order quantities $Q(I \mid S I)$ and $Q(I \mid S S)$ in terms of second-order state variables. The following relations between the pair variables such as $[S I]$ and the local neighborhood variables such as $Q(S \mid I)$ will be useful:

$$
\begin{aligned}
Q(i \mid j) & =\frac{[i j]}{[j]} \\
Q(i \mid j k) & =\frac{[i j k]}{[j k]}
\end{aligned} \quad i \neq k
$$

where $i, j$ and $k$ denote either a susceptible $(S)$ or an infected $(I)$ individual, and where $[i j k]$ is the number of triples (of three connected, distinct individuals of type $i, j$, and $k)$.

\subsection{Ordinary pair approximation}

The moment closure is typically made by assuming that the infection status of neighbors of a given individual are conditionally independent from one another. Thus, the fact that a susceptible individual is known to have at least one infected neighbor does not change the expected number of infected individuals amongst the other $Q-1$ neighbors. Therefore

$$
\begin{aligned}
Q(I \mid S I) & \approx 1+\frac{Q-1}{Q} Q(I \mid S)=1+\frac{Q-1}{Q} \frac{[S I]}{[S]} \\
Q(I \mid S S) & \approx \frac{Q-1}{Q} Q(I \mid S)=\frac{Q-1}{Q} \frac{[S I]}{[S]}
\end{aligned}
$$


where the additive factor of 1 in Eq. (10) occurs because we know the susceptible individual already has at least one infected neighbor. This closure is known as the ordinary pair approximation (OPA) $[4,14]$. The OPA can also be derived rigorously subject to the assumption that the number of infected neighbors $Q_{x}(I)$ of the susceptible individual $x$ is binomially distributed with mean $Q(I \mid S)$ [29].

Inserting Eqs. (10) and (11) into Eqs. (4)-(6) yields the closed equations of motion for $[S I],[I I]$, and $[S S]$ under the ordinary pair approximation,

$$
\begin{aligned}
\frac{d}{d t}[S I] & =-\nu[S I]+\nu[I I]-\lambda[S I]\left(1+\frac{Q-1}{Q} \frac{[S I]}{[S]}\right)+\lambda[S S] \frac{Q-1}{Q} \frac{[S I]}{[S]}, \\
\frac{d}{d t}[I I] & =-2 \nu[I I]+2 \lambda[S I]\left(1+\frac{Q-1}{Q} \frac{[S I]}{[S]}\right) \\
\frac{d}{d t}[S S] & =2 \nu[S I]-2 \lambda[S S] \frac{Q-1}{Q} \frac{[S I]}{[S]} .
\end{aligned}
$$

Finally, since the lattice is regular, the constraint

$$
Q=2[S I]+[I I]+[S S]
$$

applies, making one of the pair equations redundant and resulting in a two-dimensional system of coupled nonlinear equations.

\subsection{Triangular pair approximation (TPA)}

A number of methods for improving the ordinary pair approximation (and in particular for dealing with the inaccuracies introduced by assuming conditional independence) have been proposed $[14,15,29-32]$. One source of inaccuracy in the OPA comes from clumping effects. In certain lattices (e.g. hexagonal lattices, and square lattices with $Q=8$ ), two neighboring individuals may have a common neighbor, thus forming a triangle of three mutually interconnection individuals. This reduces the accuracy of the conditional independence assumption. Here we describe an alternative pair approximation that was developed to correct for clumping [18,29]. We will refer to it as the triangular pair approximation (TPA). 
Let $\phi$ be the ratio of the number of triangles in the lattice to the number of triples. For example, for a hexagonal lattice we have $\phi=0.2$ (each triangle has three of its 'own' triples, and 'shares' twelve triples with six other neighboring triangles, hence $\phi=1 /(3+12 / 6)=1 / 5)$. For a square lattice with $Q=8$ (the Moore neighborhood) we have $\phi=8 / 84$. For a square lattice with $Q=4$ (the von Neumann neighborhood), and for a triangular lattice with $Q=3$, we have $\phi=0$. Larger values of $\phi$ correspond to networks with greater clumping. The TPA makes the approximation

$$
[i j k] \approx \frac{Q-1}{Q} \frac{[i j][j k]}{[j]}\left(1-\phi+\phi\left\{\frac{N[i k]}{Q[i][k]}\right\}\right) .
$$

For the case $\phi=0$ this closure is equivalent to the OPA. Under the TPA, it can be shown that

$$
\begin{aligned}
& Q(I \mid S I) \approx 1+(1-\phi) \frac{Q-1}{Q} \frac{[S I]}{[S]}+\phi \frac{Q-1}{Q^{2}} \frac{[S I][I I]}{[S][I]^{2}} \\
& Q(I \mid S S) \approx(1-\phi) \frac{Q-1}{Q} \frac{[S I]}{[S]}+\phi \frac{Q-1}{Q^{2}} \frac{[S I]^{2}}{[S]^{2}[I]}
\end{aligned}
$$

\subsection{Ordinary triple approximation (OTA)}

Instead of closing the equations of motion at the level of pairs by approximating third-order quantities in terms of second-order quantities, it is also possible to close the equations at the level of triples by expressing fourth-order quantities in terms of third-order quantities $[4,21]$. Here we derive such a triple approximation.

A triple is defined as three connected, distinct individuals. Here we derive equations for the triples $[I I I]$ (twice the number of infected-infected-infected triples), [SIS] (twice the number of susceptible-infected-susceptible triples), $[I S I]$ (twice the number of infected-susceptible-infected triples), [SSS] (twice the number of susceptiblesusceptible-susceptible triples), [IIS] (number of infected-infected-susceptible triples) and $[I S S]$ (number of infected-susceptible-susceptible triples). The equations of mo- 
tion for the triples are

$$
\begin{aligned}
\frac{d}{d t}[I I I]= & -3 \nu+2 \lambda[I I S] Q(I \mid S I I)+\lambda[I S I] Q\left(I \mid S<_{I}^{I}\right), \\
\frac{d}{d t}[I I S]= & \nu[I I I]-2 \nu[I I S]+\lambda[I S S] Q\left(I \mid S<_{S}^{I}\right)+\lambda[S I S] Q(I \mid S I S) \\
& -\lambda[I I S] Q(I \mid S I I), \\
\frac{d}{d t}[I S I]= & \nu[I I I]-2 \nu[I S I]+2 \lambda[I S S] Q(I \mid S S I)-\lambda[I S I] Q\left(I \mid S<_{I}^{I}\right), \\
\frac{d}{d t}[S I S]= & 2 \nu[I I S]-\nu[S I S]+\lambda[S S S] Q\left(I \mid S<_{S}^{S}\right)-2 \lambda[S I S] Q(I \mid S I S), \\
\frac{d}{d t}[I S S]= & \nu[I I S]+\nu[I S I]-\nu[I S S]+\lambda[S S S] Q(I \mid S S S) \\
& -\lambda[I S S] Q\left(I \mid S<_{S}^{I}\right)-\lambda[I S S] Q(I \mid S S I), \\
\frac{d}{d t}[S S S]= & 2 \nu[I S S]+\nu[S I S]-2 \lambda[S S S] Q(I \mid S S S) \\
& -\lambda[S S S] Q\left(I \mid S<_{S}^{S}\right),
\end{aligned}
$$

where $Q(I \mid S S S)$ is the average number of infected neighbors of a susceptible that has at least one susceptible neighbor who in turn has a (different) susceptible neighbor (and likewise for $Q(I \mid S I S), Q(I \mid S I I)$ and $Q(I \mid S S I))$, and where $Q\left(I \mid S<_{S}^{I}\right)$ is the average number of infected neighbors of a susceptible that has at least one infected neighbour and at least one susceptible neighbor (and likewise for $Q(I \mid S<I)$ and $\left.Q\left(I \mid S<{ }_{S}^{S}\right)\right)$. The corresponding equations for pairs, now without moment closure approximation, are

$$
\begin{aligned}
\frac{d}{d t}[S I] & =-\nu[S I]+\nu[I I]-\lambda[I S I]-\lambda[S I]+\lambda[I S S] \\
\frac{d}{d t}[I I] & =-2 \nu[I I]+2 \lambda[S I]+2 \lambda[I S I] \\
\frac{d}{d t}[S S] & =2 \nu[S I]-2 \lambda[I S S]
\end{aligned}
$$

With the pair constraint (Eq. (15)) and a similar constraint for the number of triples,

$$
2 T=[I I I]+[S S S]+[I S I]+[S I S]+2[I I S]+2[I S S]
$$

the system of equations (18)-(26) can be reduced to seven dimensions. We approximated the fourth-order quantities that appear in Eqs. (18)-(23) in terms of third-order 
quantities as follows:

$$
\begin{aligned}
Q(I \mid S I I) & \approx Q(I \mid S I)=1+\frac{[I S I]}{[S I]} \\
Q(I \mid S I S) & \approx Q(I \mid S I)=1+\frac{[I S I]}{[S I]} \\
Q(I \mid S S I) & \approx Q(I \mid S S)=\frac{[I S S]}{[S S]} \\
Q(I \mid S S S) & \approx Q(I \mid S S)=\frac{[I S S]}{[S S]} \\
Q\left(I \mid S<_{I}^{I}\right) & \approx Q(I \mid S I)=1+\frac{[I S I]}{[S I]} \\
Q\left(I \mid S<_{S}^{I}\right) & \approx Q(I \mid S S)=\frac{[I S S]}{[S S]} \\
Q\left(I \mid S<_{S}^{S}\right) & \approx Q(I \mid S S)=\frac{[I S S]}{[S S]}
\end{aligned}
$$

and we refer to this as the ordinary triple approximation (OTA). Other triple closures are possible, however this particular closure was found to give the most accurate $\mathcal{R}_{0}$ values, despite some anomalies in time series of $C_{S I}$ (see Fig. 5 caption).

\subsection{Invasory pair approximation (IVPA)}

During the early stages of epidemic invasion in a spatial population, infected individuals are clustered together in areas of the lattice where the first infected cases were introduced. We refer to these infected areas as invading clusters, since their spatial extent grows over time. More specifically, an individual is considered to be part of an invading cluster if infected, or if susceptible and neighboring an infected individual. Likewise, a susceptible-susceptible pair is in the invading cluster if one of the two susceptible individuals neighbors an infected individual. All infected-infected and infected-susceptible pairs are also defined to be part of the invading cluster.

When the epidemic is initially restricted to small parts of the lattice, such that $[S I] \ll[S]$, the ordinary pair approximation (Eq. (10)) predicts $Q(I \mid S I) \approx 1$.

However, the conditional part of the expression $Q(I \mid S I)$ specifies that the susceptible individual has at least one infected neighbor, and since infected individuals are 
concentrated within invading clusters, we should expect that $Q(I \mid S I)>1$ during the early stages of invasion. In effect, the OPA does not take into account the fact that infected individuals are clustered together during the early stages of invasion; rather, it assumes that susceptible-infected pairs are uniformly distributed across the lattice. One way of circumventing this problem is simply to retain $[I S I]$ as a state variable (exploiting the identify $Q(I \mid S I)=1+[I S I] /[S I]$ ) and to write down a triple approximation $[4,21]$, such as the OTA of Section 3.3. However triple approximations, though usually more accurate than pair approximations [4,21] tend to be less tractable.

To address this problem without resorting to a triple approximation, we replace the global variables $[S I]$ and $[S]$ in Eq. (10) by the variables $[S I]_{i n v}$ (number of susceptible-infected pairs in the invading cluster) and $[S]_{i n v}$ (number of susceptible individuals in the invading cluster) respectively. In this way, we effectively retain the information in the conditional part of the expression $Q(I \mid S I)$ which tells us that the susceptible individual concerned is part of an invading cluster. Note that $[S I]_{i n v}=[S I],[I I]_{i n v}=[I I]$ and $[I]_{i n v}=[I]$ by definition, but $[S]_{i n v} \ll[S]$ during the early stages of invasion. Hence

$$
Q(I \mid S I) \approx 1+\frac{Q-1}{Q} \frac{[S I]}{[S]_{i n v}}
$$

To estimate $[S]_{\text {inv }}$ we must know something about the structure of invading clusters. The epidemic dynamics inside clusters have reached a pseudo-equilibrium that is approximated by the equilibrium for the whole population. In other words, those parts of the lattice within the invading clusters have already reached a state close to the final equilibrium predicted by Eqs. (12)-(14) to occur once the infection has spread throughout the entire lattice. Hence, it is natural to exploit this phenomenon to approximate $[S]_{i n v}$.

Let $Q(S \mid I)^{p e q}$ denote the value of $Q(S \mid I)$ at the pseudo-equilibrium inside an invading cluster. From Eq. (9) we have $[S I]=[I] Q(S \mid I)$. Substituting this into 
Eq. (1), setting $\frac{d}{d t}[I]=0$ and solving for $Q(S \mid I)$ yields

$$
Q(S \mid I)^{p e q}=\frac{\nu}{\lambda},
$$

which is the average number of susceptible individuals neighboring an infected individual inside the cluster. We approximate $[S]_{i n v}$ (the total number of susceptible individuals neighboring infected individuals), as the total number of infected individuals $([I])$ times the expected number of susceptible neighbors of an infected individual inside the invading cluster $\left(Q(S \mid I)^{p e q}\right)$. Hence, from Eq. (36) we obtain

$$
[S]_{i n v} \approx[I] Q(S \mid I)^{p e q}=\frac{\nu}{\lambda}[I] .
$$

Note that $[S]_{i n v} \propto[I]$, as should be expected, since the relative number of susceptible and infected individuals within an invading cluster should be almost constant at the pseudo-equilibrium. Finally, substituting Eq. (37) into Eq. (35) yields the IVPA approximation for $Q(I \mid S I)$,

$$
Q(I \mid S I) \approx 1+\frac{Q-1}{Q} \frac{\lambda}{\nu} \frac{[S I]}{[I]} .
$$

Note that it is not necessary to use $[S]_{i n v}$ to alter the closure for $Q(I \mid S S)$, since $[S S] Q(I \mid S S)=[I S S]=[S S]_{i n v} Q(I \mid S S)_{i n v}$. Therefore, using the OPA assumption that

$$
Q(I \mid S S) \approx \frac{Q-1}{Q} \frac{[S I]}{[S]}
$$

does not introduce the kind of error that Eq. (10) introduces, and we can retain Eq. (39) to approximate $Q(I \mid S S)$. Eqs. (38) and (39) constitute the IVPA closure.

The approximation $[S]_{i n v} \approx[I] Q(S \mid I)^{p e q}$ overestimates $[S]_{i n v}$, since susceptible individuals who are neighbors of more than one infected individual will be counted more than once. The maximum relative error thus introduced depends upon the lattice geometry: for square lattices with $Q=4$, the relative error introduced is bounded above by 2 , since for every node there are two edges. However, because $[S]_{i n v} \leq[I] Q(S \mid I)^{p e q} \ll[S]$, assuming $[S]_{i n v} \approx[I] Q(S \mid I)^{p e q}$ still improves upon 
the ordinary pair approximation by orders of magnitude. As the infection spreads throughout the network, it eventually occurs that $[S]_{i n v} \approx[I] Q(S \mid I)^{p e q}$ exceeds $[S]$. Beyond this point the IVPA becomes less accurate than the OPA. Since $[S]_{\text {inv }} \approx \frac{\nu}{\lambda}[I]$, the requirement that $[S]_{i n v}<[S]$ translates into the condition on prevalence

$$
\frac{[I]}{N}<\frac{\lambda}{\lambda+\nu}
$$

The existence of a pseudo-equilibrium state within invading clusters has also been exploited previously in a pair-edge approximation [30]. Whereas the IVPA is a general moment closure approximation that provides equations of motion for pairs in any lattice geometry, the pair-edge approximation is not a moment closure approximation per se, but rather is concerned with estimating the speed of wavefront propagation of in the contact process for one-dimensional and approximate two-dimensional lattices. A further difference is that the IVPA requires the assumption of Eq. (35), whereas the pair-edge approximation exploits a distinction between slow and fast variables to estimate wavespeed (in Section 4 we will also use this distinction in order derive $\mathcal{R}_{0}$ ).

As a result, the pair-edge estimate of the critical point for the contact process [30] is more accurate than the IVPA prediction (Fig. 4).

In the next section, we show that the IVPA predicts the basic reproductive number $\mathcal{R}_{0}$ more accurately than the OPA, TPA and OTA, as well as yielding simpler closedform expressions for $\mathcal{R}_{0}$ than either the TPA or the OTA.

\section{Calculation of $\mathcal{R}_{0}$ using moment closure tech- niques}

Under the mean-field approximation, $\mathcal{R}_{0}=\beta / \nu[27]$. This overestimates the true $\mathcal{R}_{0}$ in a spatially structured population because it does not take into account the slowing effects of spatially localized transmission. The moment closure technique used to derive $\mathcal{R}_{0}$ relies upon the pair approximation's capacity to implicitly capture 
the time evolution of local spatial structure. The technique has been applied to an SIR epidemic on a lattice [3,9] and to an SIS epidemic on a dynamic, irregular network [17] using the OPA, as well as to lattice ecological models [4,30]. Here we apply the technique to an SIS epidemic on a lattice, under the OPA, TPA, OTA and IVPA.

We start by rewriting the equation of motion for $[I]$ in terms of the susceptibleinfected correlation function $C_{S I} \equiv \frac{N[S I]}{Q[S][I]}$,

$$
\begin{aligned}
\frac{d}{d t}[I] & =-\nu[I]+\lambda[S I] \\
& =[I]\left(-\nu+\lambda \frac{Q[S] C_{S I}}{N}\right) .
\end{aligned}
$$

Since $[S] \approx N$ initially, this simplifies to

$$
\frac{d}{d t}[I]=[I]\left(-\nu+\lambda Q C_{S I}\right)
$$

$R_{0}$ is commonly identified with the threshold in Eq. (42) above which $[I]$ grows and an epidemic occurs, and below which $[I]$ decreases exponentially and the disease fades out, yielding

$$
\mathcal{R}_{0}=\frac{\lambda Q C_{S I}}{\nu}=\frac{\beta C_{S I}}{\nu}
$$

Since this method of approximating $\mathcal{R}_{0}$ extrapolates from an individual's instantaneous infection rate $\beta C_{S I}$ at the minimum in $C_{S I}$ over the entire interval of the individual's expected duration of infectiousness $(1 / \nu)$, we describe this method as an example of a rate-based approximation to $\mathcal{R}_{0}$.

When the infectious disease is first introduced, a small number of individuals are chosen randomly and inoculated with the disease, hence $C_{S I} \approx 1$. Thereafter $C_{S I}$ decreases as infected individuals begin to transmit the infection to their neighbors. The resulting clustering of infecteds decreases the rate of spread of the epidemic, since some effective contacts will be 'wasted' on individuals who are already infected. Therefore the epidemic may die out at this stage, even when the mean-field $\mathcal{R}_{0}$ predicts a successful invasion. At the beginning of the epidemic, we see from simulations 
of the lattice model (Fig. 1) and analytical arguments [9] that $C_{S I}$ reaches a locally minimum value $C_{S I}^{\min }$ where $\frac{d}{d t} C_{S I}=0$. It is the structure of invading clusters at $C_{S I}^{\min }$ that actually determines the ultimate success or failure of the invasion $[3,9,17]$. The number of new infecteds that arise is determined by the frequency of susceptibleinfected pairs, and so the epidemic must be able to spread when $C_{S I}$ is minimal, if it is to invade the remainder of the population.

Accordingly we need to calculate $C_{S I}^{m i n}$ and substitute into Eq. (43) to obtain a rate-based approximation to the basic reproductive number $\mathcal{R}_{0}$ on a regular lattice. Although some stochastic effects are ignored by this deterministic analysis, and generally serve to slow the epidemic still further, the pair approximation $\mathcal{R}_{0}$ nonetheless captures important aspects of spatial structure.

The equation of motion for $C_{S I}$ is

$$
\frac{d}{d t} C_{S I}=\frac{N}{Q}\left[\frac{1}{[S][I]} \frac{d}{d t}[S I]+\frac{[S I]}{[S][I]}\left(-\frac{1}{[I]} \frac{d}{d t}[I]-\frac{1}{[S]} \frac{d}{d t}[S]\right)\right]
$$

Substituting the equation of motion for $[I]$ (Eq. (2)) and the OPA equation of motion for $[S I]($ Eq. (12)) into Eq. (44) yields

$$
\begin{aligned}
\frac{d}{d t} C_{S I}= & \nu \frac{N}{[S]}\left(\frac{[I] C_{I I}}{N}\right)-\lambda C_{S I}-\lambda(Q-1) \frac{[I]}{N} C_{S I}^{2}+\lambda(Q-1) \frac{[S]}{N} C_{S S} C_{S I} \\
& -\lambda Q \frac{[S]}{N} C_{S I}^{2}-\nu \frac{[I]}{[S]} C_{S I}+\lambda Q \frac{[I]}{N} C_{S I}^{2} .
\end{aligned}
$$

In the early stages of invasion we can assume that $[S] \approx N,[I] / N \ll 1$, and $[S I] / Q N \ll 1$, hence

$$
\frac{d}{d t} C_{S I}=\nu\left(\frac{[I] C_{I I}}{N}\right)-\lambda C_{S I}+\lambda(Q-1) C_{S I}-\lambda Q C_{S I}^{2}
$$

(We note in passing that Eq. (46) is identical to Eq. (A4) of the pair-edge approximation of Ellner et al. [30], with necessary replacement of symbols.) The quantity $\left([I] C_{I I} / N\right)$, like the other terms in Eq. (44), is of order 1 since $\left([I] C_{I I} / N\right)=[I] \frac{[I I]}{Q[I]^{2}}=$ 
$\frac{Q(I \mid I)}{Q}$. In a similar fashion we derive the equation of motion for $\left([I] C_{I I} / N\right)$,

$$
\begin{aligned}
\frac{d}{d t}\left(\frac{[I] C_{I I}}{N}\right)= & \frac{1}{Q}\left(\frac{1}{[I]} \frac{d}{d t}[I I]-\frac{[I I]}{[I]^{2}} \frac{d}{d t}[I]\right) \\
= & -\nu\left(\frac{[I] C_{I I}}{N}\right)+2 \lambda C_{S I} \frac{[S]}{N}+2 \lambda(Q-1) C_{S I}^{2} \frac{[S][I]}{N^{2}} \\
& -\lambda Q C_{S I}\left(\frac{[I] C_{I I}}{N}\right) \frac{[S]}{N} .
\end{aligned}
$$

When $[S] \approx N$ and $[I] / N \ll 1$ this becomes

$$
\frac{d}{d t}\left(\frac{[I] C_{I I}}{N}\right)=-\nu\left(\frac{[I] C_{I I}}{N}\right)+2 \lambda C_{S I}-\lambda Q C_{S I}\left(\frac{[I] C_{I I}}{N}\right)
$$

We obtain $C_{S I}^{\min }$ by setting the left hand sides of Eqs. (46) and (48) equal to zero and solving simultaneously for $C_{S I}$ and $\left([I] C_{I I} / N\right)$ :

$$
\begin{aligned}
C_{S I}^{\min } & =\frac{1}{2} \frac{-\nu-2 \lambda+\lambda Q+\sqrt{\nu^{2}+4 \nu \lambda+2 \lambda Q \nu+4 \lambda^{2}-4 \lambda^{2} Q+\lambda^{2} Q^{2}}}{\lambda Q} \\
\left(\frac{[I] C_{I I}}{N}\right)^{\min } & =1-C_{S I}^{p e q} .
\end{aligned}
$$

Substituting Eq. (49) into Eq. (43) (after using Eq. (3) to re-express Eq. (49) in terms of $\beta, Q$ and $\nu$ ) yields the expression for $\mathcal{R}_{0}$ as derived under the OPA:

$$
\mathcal{R}_{0}=\frac{\beta(Q-2)}{2 \nu Q}-\frac{1}{2}+\frac{1}{2} \sqrt{\left(\frac{\beta(Q-2)}{\nu Q}+1\right)^{2}+8 \frac{\beta}{Q \nu}} .
$$

Following the same procedure but using the IVPA (see Appendix B) yields

$$
\begin{aligned}
\mathcal{R}_{0}= & \frac{\sqrt{\beta^{2}\left(Q^{4}+2 Q^{3}-5 Q^{2}+2 Q+1\right)+2 Q^{2} \beta \nu\left(Q^{2}+Q+1\right)+Q^{4} \nu^{2}}}{2\left(\beta Q-\beta+\nu Q^{2}\right)} \\
& +\frac{-\beta Q-\beta-\nu Q^{2}+\beta Q^{2}}{2\left(\beta Q-\beta+\nu Q^{2}\right)}
\end{aligned}
$$

In the mean-field limit, $Q=N-1$ and $N \rightarrow \infty$ while $\beta$ and $\nu$ remain constant, and we recover the mean-field expression $\mathcal{R}_{0}=\beta / \nu$ from both OPA and the IVPA expressions for $\mathcal{R}_{0}$. However further comparisons reveal important differences between the OPA and the IVPA expressions. For example, as $\beta \rightarrow \infty$ while $Q$ and $\nu$ remain constant, the OPA predicts

$$
\mathcal{R}_{0} \rightarrow \infty
$$


whereas the IVPA predicts

$$
\mathcal{R}_{0} \rightarrow \frac{-Q-1+Q^{2}+\sqrt{Q^{4}+2 Q^{3}-5 Q^{2}+2 Q+1}}{2(Q-1)} .
$$

However, as mentioned above, $\mathcal{R}_{0}$ on a lattice should be bounded above in the limit $\beta \rightarrow \infty$. To see this, assume first that, for a given value of $\beta$, the value of $\mathcal{R}_{0}$ decreases as $\nu$ increases. Secondly, observe that for $\nu=0$, an infected individual can infect no more than $Q-1$ neighbors, none of whom ever recover (the individual became infected through one of his or her neighbors, so no more than $Q-1$ neighbors can be susceptible). Hence, when $\nu=0, \mathcal{R}_{0} \leq Q-1$. For any $\nu>0$, it follows from the first assumption that $\mathcal{R}_{0}<Q-1$. Thus $\mathcal{R}_{0} \leq Q-1$ for all $\beta$ and $\nu$.

Although the OPA $\mathcal{R}_{0}$ grows unboundedly, the IVPA $\mathcal{R}_{0}$ exhibits an upper bound close to the correct value: if we assume that $\mathcal{R}_{0}$ increases monotonically in $Q$ (which is reasonable, since the slowing effects of space decrease as interactions become less localized), then an upper bound on $\mathcal{R}_{0}$ under the IVPA can be obtained by taking the limit $Q \rightarrow \infty$ in Eq. (53), yielding

$$
\mathcal{R}_{0}<Q+\frac{1}{2}
$$

The procedure can be repeated for the TPA and the OTA. The resulting expression for the TPA is unwieldy and improves only slightly on the OPA expression: the predicted $\mathcal{R}_{0}$ values still increases unboundedly as $\beta \rightarrow \infty$. The OTA is even more difficult to analyze, however numerical results indicate that the OTA prediction of $R_{0}$ is bounded. The OTA predicts higher (less accurate) $\mathcal{R}_{0}$ values than the IVPA for all values of $\beta$.

Fig. 2 summarizes these results and benchmarks them against stochastic individualbased simulations of an SIS epidemic on a hexagonal lattice. The IVPA predicts the simulation values of $\mathcal{R}_{0}$ most accurately out of all the deterministic approximations considered, for all values of the transmission rate $\beta$. Defining $\mathcal{R}_{0}$ for an individual-based lattice model such that it is comparable to the rate-based $\mathcal{R}_{0}$ expressions computed using moment closure techniques is nontrivial. The rate-based $\mathcal{R}_{0}$ 
estimates computed above extrapolate from the instantaneous infection rates at the time $t_{\min }$ (the time at which $C_{S I}$ achieves its minimum value $C_{S I}^{\min }$ ). However, from an individual-based perspective, $\mathcal{R}_{0}$ is the average number of secondary infections produced by an infected individual over the entire duration of his or her infectiousness (not just at a single point in time $t_{\text {min }}$ ). The individual-based definition of $\mathcal{R}_{0}$ is really an integration of infection rates over a time interval of average length $1 / \nu$, and this interval is generally large compared to $t_{m i n}$. Therefore it is not possible to compute an individual-based $\mathcal{R}_{0}$ by looking only at instantaneous infection rates at $t_{\min }$.

Hence, for the lattice model simulations in Fig. 2, we defined $\mathcal{R}_{0}$ as the average number of secondary infections produced by an infected individual who was infected at time $t_{\mathcal{R}_{0}} \equiv \max \left(t_{m i n}-1 / 2 \nu, 0\right)$, where $1 / 2 \nu$ is half the mean duration of infectiousness. Hence $\mathcal{R}_{0}$ is the average number of secondary infections produced by an individual who, on average, should have been halfway through their bout of infectiousness at time $t_{\text {min }}$, or if $t_{\min }>1 / 2 \nu$, who was infected at the start of the simulation. Fig. 2 shows values of $R_{0}$ for the individual-based simulation according to this definition, and also shows a maximal $\mathcal{R}_{0}$ value (the number of secondary infections produced by an individual who was infected at $t=0$ ). For $\beta \gtrsim 1.6, \mathcal{R}_{0}$ and maximal $\mathcal{R}_{0}$ are identical since $t_{\min }<1 / 2 \nu$ for sufficiently large $\beta$.

Notably, in Fig. 2, all moment closure approximations converge to the simulation values of $\mathcal{R}_{0}$, thus becoming more accurate as $\beta$ approaches the critical point $\mathcal{R}_{0}=1$. By comparison, moment closure approximations usually become less accurate in their predictions of equilibrium quantities (such as $[I]$ ) as they approach the critical point $[4,21]$. This puzzling difference may partly be due to the the way that rate-based approximations to $\mathcal{R}_{0}($ e.g., Section 4$)$ are computed. $\mathcal{R}_{0}$ as an individualbased quantity is computed over the entire interval of an individuals infectiousness. However rate-based approximations to $\mathcal{R}_{0}$ extrapolate from an individuals instantaneous infection rate at the local minimum in $C_{S I}$ across the entire interval of his or 
her infectiousness (e.g., Eq. (43)). When $t_{\text {min }} \gg 1 / \nu$ (as occurs for small $\beta$ ), this extrapolation not introduce much error. However, as $\beta$ increases, $t_{\text {min }}$ becomes small compared to $1 / \nu$, so the error caused by extrapolating the instantaneous infection rate at $C_{S I}^{\min }$ to the entire interval of infectiousness will increase (although the error is much less for the OTA and IVPA than for the other moment closure approximations).

Fig. 3 illustrates how the moment closure expressions for $\mathcal{R}_{0}$ capture the slowing effects of space on disease transmission: as the neighborhood size $Q$ decreases, $\mathcal{R}_{0}$ also decreases. This behavior agrees with the predictions of other spatial models. As $Q$ increases, all PA expressions for $\mathcal{R}_{0}$ approach the mean-field approximation $\mathcal{R}_{0}=\beta / \nu$. Spatial structure has the largest slowing effect under the IVPA. Also, as expected, the pair approximations converge to one another as $Q \rightarrow 2$, since conditional independence between the infection states of an individual's neighbors holds true in a linear lattice.

Fig. 4 compares $\nu_{c}$ (the value of the recovery rate $\nu$ at the critical point $\mathcal{R}_{0}=1$ ) for the stochastic individual-based simulations of the lattice model, and the TPA, OPA, OTA, IVPA and mean-field approximations. For $\nu<\nu_{c}$ the disease becomes endemic whereas for $\nu \geq \nu_{c}$ the population is disease-free. For the mean-field and moment closure approximations for $\mathcal{R}_{0}$, the values of $\nu_{c}$ are obtained by setting $\mathcal{R}_{0}=1$ and solving for $\nu$. The TPA $\mathcal{R}_{0}$ predicts the value of $\nu_{c}$ in lattice model simulations most accurately, and all moment closure approximations are significantly more accurate than the mean-field prediction. Interestingly, the OPA and IVPA predictions of the threshold are identical.

Fig. 5 depicts the time evolution of global variables $([I],[S I])$, and local spatial variables $\left(Q(S \mid I)\right.$ and $\left.C_{S I}\right)$ in the lattice model simulations and the deterministic approximations. These time series give us a detailed look at how the deterministic approximations capture, or fail to capture, various aspects of invasion in a spatially structured population. We observe that moment closure approximations do poorly in predicting the time evolution of most global and local spatial quantities during 
invasion, even when they can predict equilibrium values with high accuracy. This suggests that caution should be exercised when applying moment closure approximations to study spatial invasion, even when the approximations accurately predict the final states of the system. However, there is an important exception to this general pattern: the IVPA accurately predicts $C_{S I}^{\min }$ (for the parameter values in Fig. 5 as

well as other parameter values). An accurate estimate of $C_{S I}^{\min }$ is needed to compute $\mathcal{R}_{0}$, and this is why the IVPA does best in Fig. 2 . The IVPA underestimates $t_{\text {min }}$ by a factor of two, although $t_{\text {min }}$ is not needed to compute $\mathcal{R}_{0}$. The IVPA also predicts the time evolution of $[I]$ more accurately for $t \lesssim 10$ days, although it does significantly worse than other approximations in predicting the equilibrium values, and should not be used to approximate equilibrium states. The source of failure of the IVPA for predicting equilibrium states relates to the fact that the approximation used for $[S]_{i n v}$ exceeds $[S]$ when the prevalence is sufficiently large (Eq. (40) gives the conditions for this to occur.)

\section{Discussion and Conclusions}

The literature on pair approximations usually emphasizes the study of equilibrium states, but here we have focused on invasion dynamics. We used a previously developed moment closure technique $[3,9]$ to derive approximate expressions for $\mathcal{R}_{0}$ on a regular lattice. We compared these $\mathcal{R}_{0}$ expressions as derived under ordinary, triangular and invasory pair approximations, as well as a triple approximation, to $\mathcal{R}_{0}$ values from simulations of the corresponding lattice model. We found that the invasory pair approximation (IVPA) expression for $\mathcal{R}_{0}$ introduced in this paper was the most accurate of the moment closure approximations, while also providing a relatively simple closed-form expression for $\mathcal{R}_{0}$. Moreover, the IVPA should be applicable to a broad range of spatial population models since it relies upon characteristics of invasion that are common to all spatially structured populations (in particular, the existence of 
a pseudo-equilibrium state within the invading clusters and the fact that infections are usually restricted to small areas of the lattice in the initial stages of invasion). Because most pair approximations do not take into account these characteristics of invasion, we suggest that the large qualitative and quantitative inaccuracies exhibited by the ordinary pair approximation (OPA) and triangular pair approximation (TPA) will be common to many pair approximations. Such errors have also been observed in pair approximations for epidemic models in continuous space [33].

Although the IVPA is most accurate during invasion, the OPA and TPA predict the equilibrium state more accurately than the IVPA. Future work may explore the possibility of combining these two approximations such that the IVPA is weighted more heavily during the early stages of invasion, and the OPA more heavily in later stages, producing an optimal combined pair approximation (e.g., Ref. [15]). The significant error of the OPA during invasion, even for parameters where the OPA does extremely well in predicting equilibrium values (see Fig. 5 for instance), underscores the danger in applying pair approximations benchmarked against lattice simulation equilibria to transient dynamics.

Even when quantitative agreement of pair approximations with lattice model simulations is not perfect, their qualitative accuracy may be useful. Pair approximations capture aspects of spatial dynamics that mean-field equations are categorically unable to. Hence, they are useful for illustrating general principles and providing a general framework for thinking about spatial population dynamics. For instance, all three pair approximations predict the initial decline in $C_{S I}$ and the existence of a pseudoequilibrium in $C_{S I}$. These features cannot be captured by a mean-field approximation, for which $C_{S I}=1$ throughout.

Pair approximations constitute a useful and widely applicable technique for studying spatial models. Understanding the time evolution of local spatial structure and deriving closed-form expressions for a 'spatial' $\mathcal{R}_{0}$ are valuable for a better understanding of invasion in spatially structured populations. The application of pair 
approximations to ecological and epidemiological systems is still young relative to their applications in physics and chemistry. Future work may further hone the accuracy of pair approximations for biological systems, and draw on pair approximation methodologies developed for applications in physics and chemistry. This is especially important given the frequent occurrence of invasions in real-world spatial ecological and epidemiological systems.

\section{Acknowledgments}

CTB is grateful to the Natural Sciences and Engineering Research Council (NSERC) and the Canadian Institutes of Health Research (CIHR) for funding support, and to David Rand for interesting discussions. 


\section{References}

[1] R. Durrett, S.A. Levin, The importance of being discrete (and spatial), Theor. Pop. Biol. 46 (1994) 363-394.

[2] S.A. Levin, B.T. Grenfell, A. Hastings, A.S. Perelson, Mathematical and computational challenges in population biology and ecosystems science, Science 275 (1997) 334-343.

[3] D.A. Rand, Correlation equations and pair approximations for spatial ecologies, In J. McGlade (Ed.), Advanced Ecological Theory. Principles and Applications, Blackwell Science, 1999, pages 100-142.

[4] H. Matsuda, N. Ogita, A. Sasaki, K. Sato, Statistical mechanics of population, Prog. Theor. Phys. 88 (1992) 1035-1049.

[5] M. van Baalen, D.A. Rand, The unit of selection in viscous populations and the evolution of altruism. J. Theor. Biol. 193 (1998) 631-648.

[6] D.A. Rand, M.J. Keeling, H. Wilson, Invasion, stability and evolution to criticality in spatially extended host-pathogen systems, Proc. R. Soc. Lond. B 259 (1995) 55-63.

[7] M.J. Keeling, B.T. Grenfell, Disease extinction and community size: Modeling the persistence of measeles, Science 275 (1997) 65-67.

[8] B.M. Bolker, S.W. Pacala, Spatial moment equations for plant competition: Understanding spatial strategies and the advantages of short dispersal, Am. Nat. 153 (1999) 575-602.

[9] M.J. Keeling, The effects of local spatial structure on epidemiology invasions, Proc. R. Soc. Lond. B 266 (1999) 859-867. 
[10] K. Tanaika, Paradoxical effect in a three-candidate voter model, Phys. Lett. A 176 (1993) 303-306.

[11] P. Kareiva, Space: the final frontier for ecological theory, Ecology 75 (1994) 1.

[12] H. Matsuda, Conditions for the evolution of altruism, in: Y. Ito, J.P. Brown, J. Kikkawa (Ed.), Animal Societies: Theories and Facts, Tokyo, Japanese Scientific Society Press, 1987, pages 67-80.

[13] V.S. Isham, Assessing the variability of stochastic epidemics, Math. Biosci. 107 (1991) 209-224.

[14] K. Sato, H. Matsuda, A. Sasaki, Pathogen invasion and host extinction in lattice structured populations, J. Math. Biol. 32 (1994) 251-268.

[15] J.A.N. Filipe, Hybrid closure-approximation to epidemic models, Physica A 266 (1999) 238-241.

[16] M. Boots, A. Sasaki, The evolutionary dynamics of local infection and global reproduction in host-parasite interactions, Ecol. Lett. 3 (2000) 181-185.

[17] C.T. Bauch, D.A. Rand, A moment closure model for STD transmission through a concurrent partnership network, Proc. R. Soc. Lond. B 267 (2000) 2019-2027.

[18] M. van Baalen, Pair approximations for different spatial geometries, in: U. Dieckmann, R. Law, J. A. J. Metz (Ed.), The Geometry of Ecological Interactions: Simplifying Spatial Complexity, Cambridge University Press, Cambridge, 2000.

[19] R. Law, U. Dieckmann, A dynamical system for neighborhoods in plant communities, Ecology 81 (2000) 2137-2148.

[20] N.M. Ferguson, C.A. Donnelly, R.A. Anderson, The foot-and-mouth epidemic in Great Britain: Pattern of spread and impact of interventions, Science 292 (2001) 1155-1160. 
[21] C. T. Bauch, A versatile ODE approximation to a network model for sexually transmitted diseases spread, J. Math. Biol. 45 (2002) 375-395.

[22] C.T. Bauc, A.P. Galvani, Using network models to approximate spatial pointprocess models, Math. Biosci. 184 (2003) 101-114.

[23] D.H. Brow, B.M. Bolker, The effects of disease dispersal and host clustering on the epidemic threshold in plants, Bull. Math. Biol. 66 (2004) 341-372.

[24] J.A.N. Filipe, W. Otten, G.J. Gibson, C.A. Gilligan, Inferring the dynamics of a spatial epidemic from time-series data, Bull. Math. Biol. 66 (2004) 373-392.

[25] T.E. Harris, Contact interactions on a lattice, Ann. Prob. 2 (1974) 969.

[26] G. MacDonald, The analysis of equilibrium in malaria, Trop. Dis. Bull. 49 (1992) 813-829.

[27] R.M. Anderson, R.M. May, Infectious Diseases of Humans, Oxford Science Publications, OUP, 1991.

[28] H. Heesteerbeek, $R_{0}, \mathrm{PhD}$ thesis, Centrum voor Wiskunde en Informatica, Amsterdam, 1992.

[29] A.J. Morris, Representing Spatial Interactions in Simple Ecological Models, PhD thesis, University of Warwick, 1997.

[30] S.P. Ellner, A. Sasaki, Y. Haraguchi, H. Matsuda, Speed of invasion in lattice population models: Pair-edge approximation, J. Math. Biol. 36 (1997) 469-484.

[31] J. Dushoff, Host heterogeneity and disease endemicity: a moment-based approach, Theor. Pop. Biol. 56 (1999) 325-335.

[32] C.T. Bauch, Moment Closure Approximations in Epidemiology, PhD thesis, University of Warwick, 2000. 
[33] B.M. Bolker, Analytic models for the patchy spread of plant disease, B. Math. Biol. 61 (1999) 849-874. 


\section{Figure Legends}

Fig. 1: Time evolution of $C_{S I}$, the S-I pair correlation function, in a stochastic individual-based simulation of an SIS epidemic on a hexagonal lattice, illustrating the local minimum that $C_{S I}$ achieves as invading clusters are formed. Parameters: $Q=6, \beta=0.6$ days $^{-1}, \nu=0.1$ days $^{-1}$, and $N=10^{6}$. Initial conditions: $1 \%$ of individuals were chosen at random and infected.

Fig. 2: Basic reproductive number $\mathcal{R}_{0}$ as predicted by the mean-field, OPA, TPA, OTA, and IVPA, compared to $\mathcal{R}_{0}$ values from stochastic individual-based simulations of the lattice model (IBM) as a function of the transmission rate $\beta$. Other parameters: $\nu=0.1$ days $^{-1}, Q=6$, and $\phi=0.2$ for the TPA. The horizontal line represents the maximum possible value for $\mathcal{R}_{0}$ on the lattice, $\mathcal{R}_{0}=Q-1$. The IBM values of $\mathcal{R}_{0}$ are significantly below the $\mathcal{R}_{0}=Q-1$ line because clustering is high in a hexagonal lattice $(\phi=0.2)$ and so an individual's neighbours are often neighbours to one another as well. See Section 4 for details on the definition of $\mathcal{R}_{0}$ and $\mathcal{R}_{0}$ maximal for the IBM.

Fig. 3: Basic reproductive number $\mathcal{R}_{0}$ as predicted by the mean-field, OPA, TPA, OTA and IVPA, as a function of the neighborhood size $Q$. This shows how the pair approximations diverge from the mean-field approximation and converge to one another as $Q \rightarrow 2$, and how pair approximations capture the slowing effects of spatial localization. Other parameters are $\nu=0.1$ days $^{-1}, \beta=0.6$ days $^{-1}$, and $\phi=0.2$ for the TPA. The OTA we use here is not valid for $Q<3$.

Fig. 4: Values of the recovery rate at the critical point $\mathcal{R}_{0}=1\left(\nu_{c}\right)$ according to the mean-field approximation, the OPA, TPA, OTA and IVPA, and stochastic individual-based simulations of the lattice model (IBM). Types of lattices used were square $(Q=4, \phi=0$ and $Q=8, \phi=0.095)$, hexagonal $(Q=6, \phi=0.2)$ and triangular $(Q=3, \phi=0)$. The transmission rate was $\beta=0.6$ days $^{-1}$. 
Fig. 5: Time evolution of the density of infected individuals $([I] / N)$, the density of susceptible-infected pairs $([S I] /(Q N / 2)), Q(S \mid I)$ and $C_{S I}$ under the meanfield, OPA, TPA, OTA and IVPA approximations, and in stochastic individual-based simulation of the lattice model (IBM). Parameters: $N=10^{6}, \nu=0.1$ days $^{-1}$, $\beta=0.6$ days $^{-1}$ and $Q=6$. For the TPA, $\phi=0.2$. Initial conditions: $1 \%$ of individuals were chosen at random and infected. For the OTA, if one assumes that $Q\left(I \mid S<_{S}^{I}\right) \approx Q(I \mid S I)$ instead of using Eq. (33), then the time series of $C_{S I}$ exhibits a trajectory with only one local minimum (which is in better qualitative agreement with simulation results) but the $R_{0}$ prediction is significantly worse. 
Figure 1

C.T. Bauch

Portrait orientation

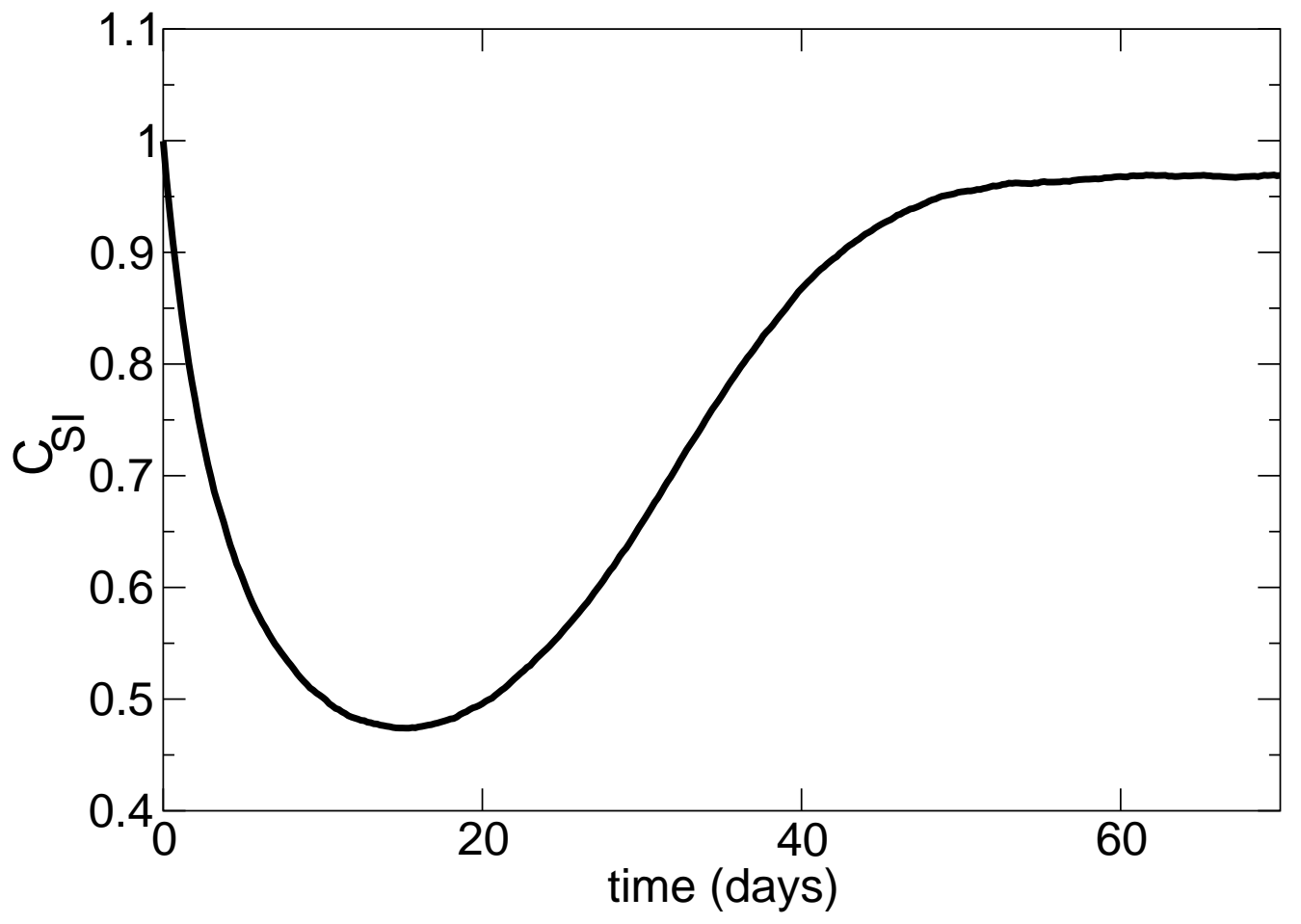


Figure 2

C.T. Bauch

Portrait orientation

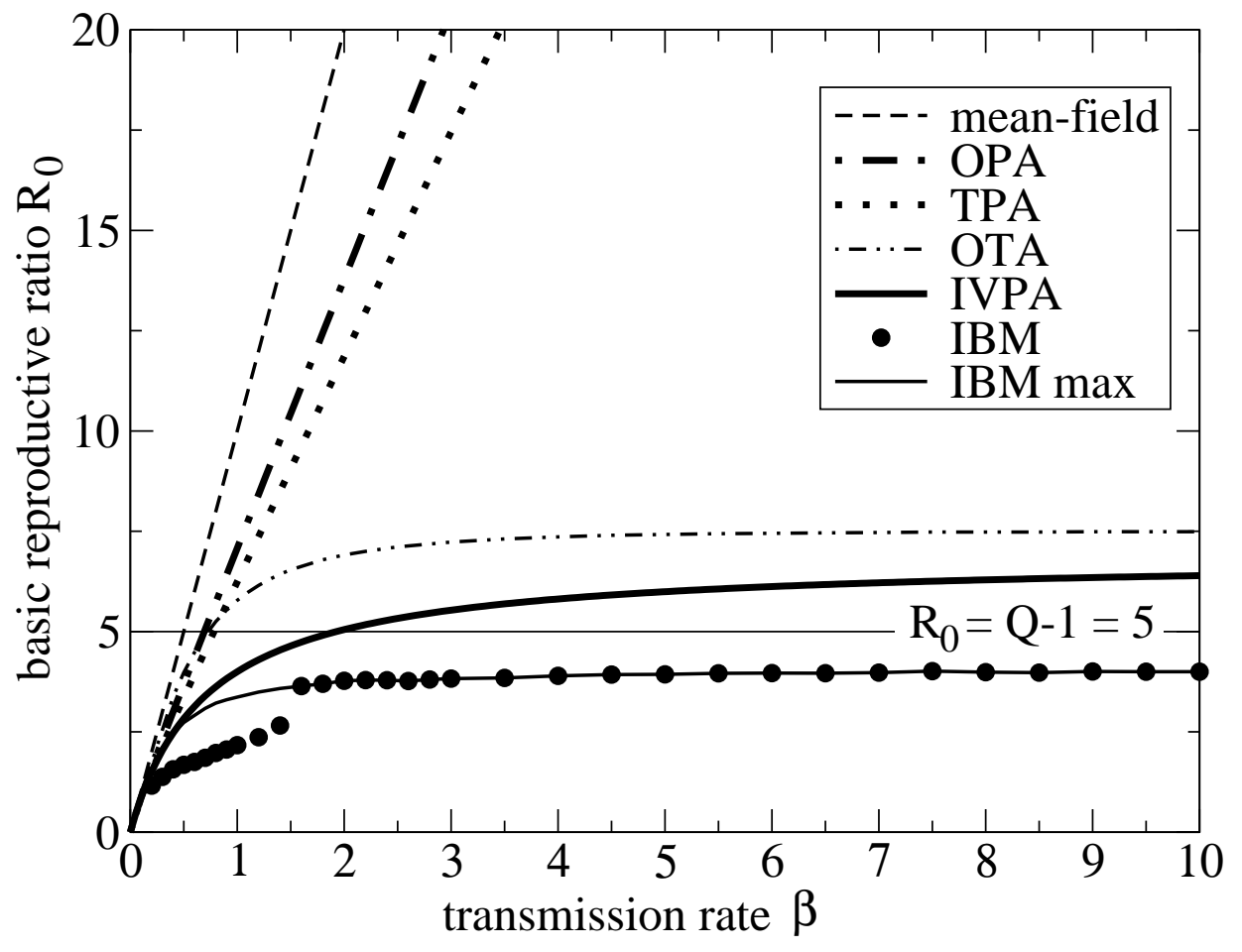


Figure 3

C.T. Bauch

Portrait orientation

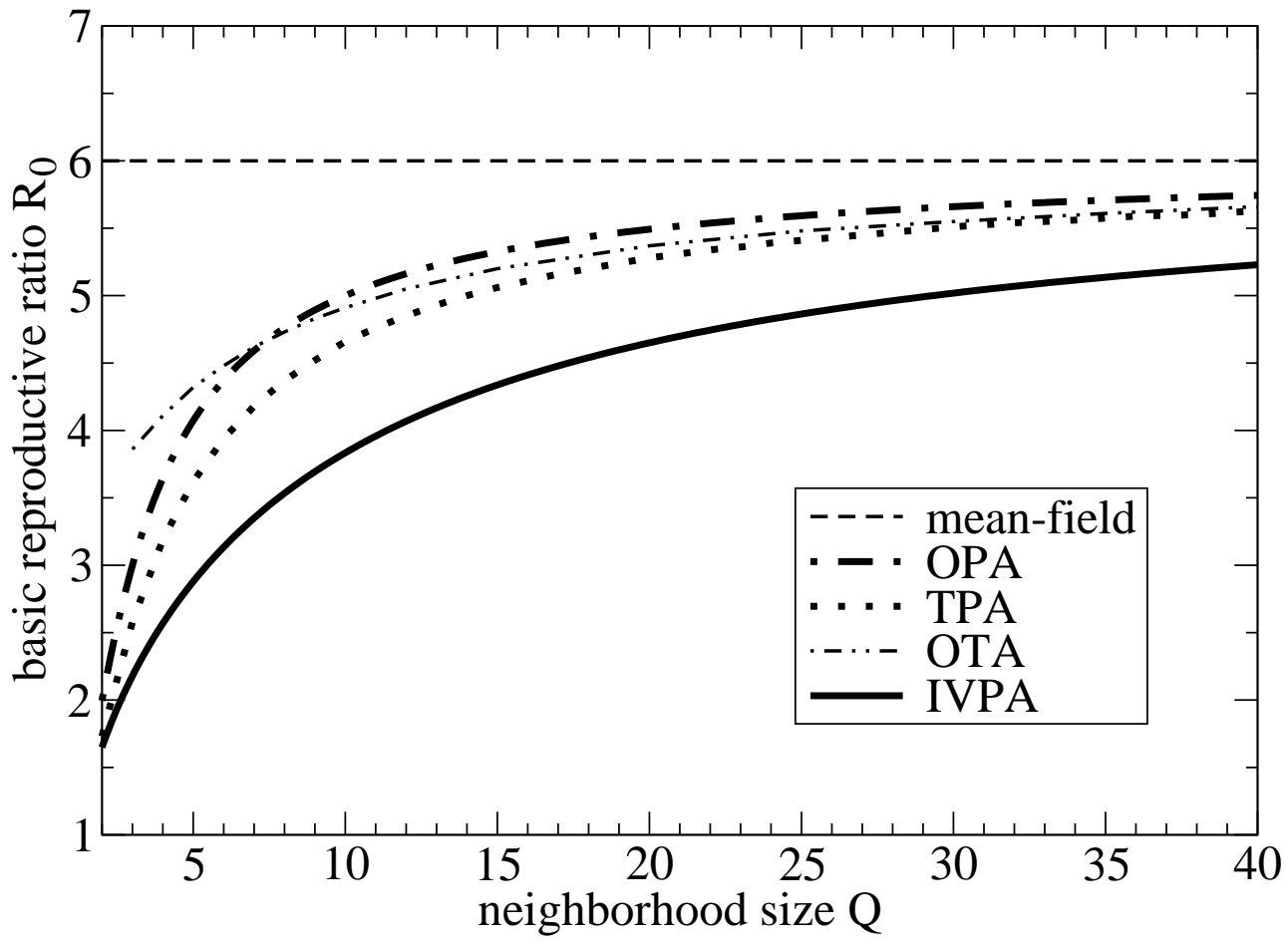


Figure 4

C.T. Bauch

Portrait orientation

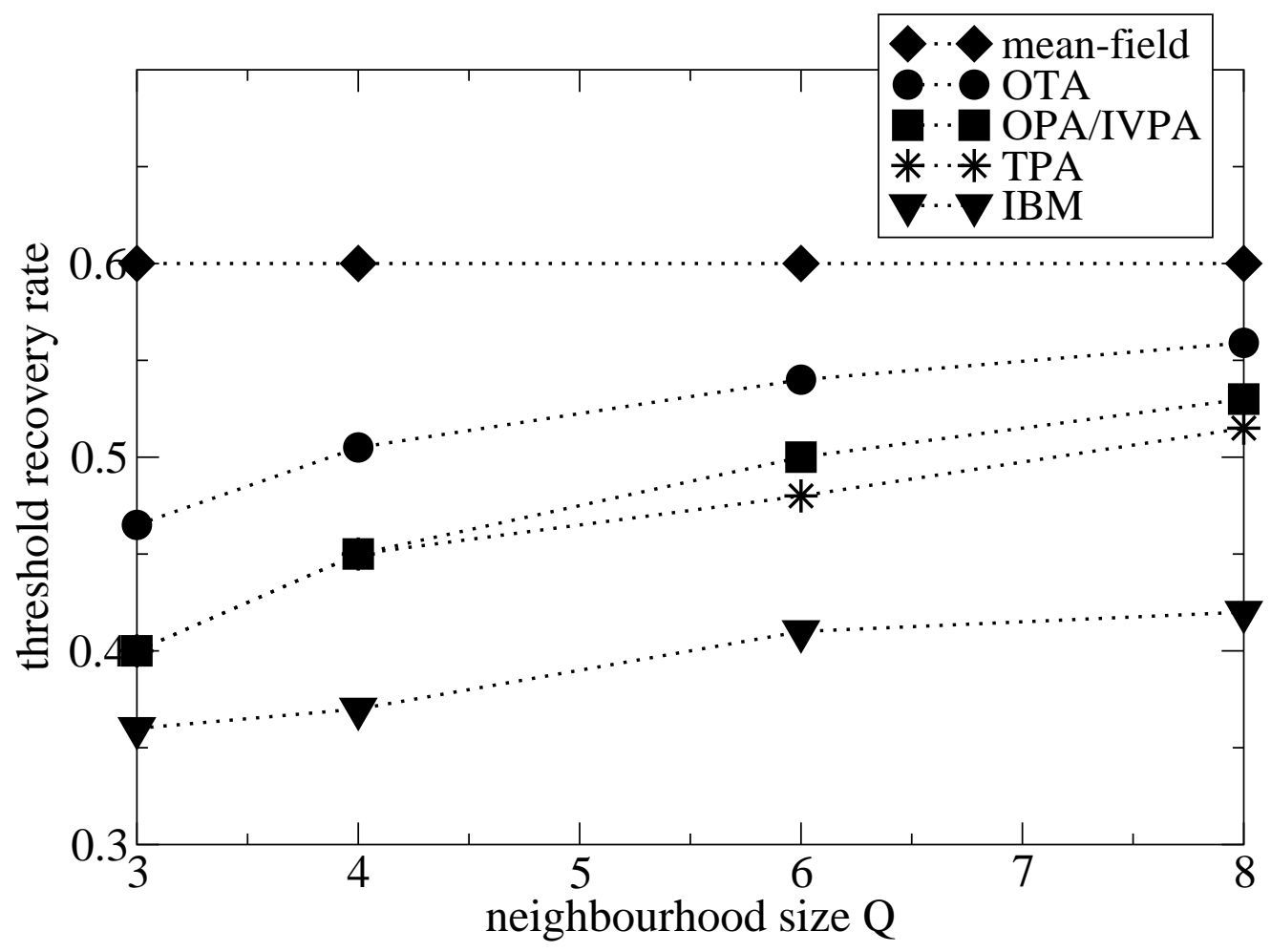


Figure 5

C.T. Bauch

Portrait orientation
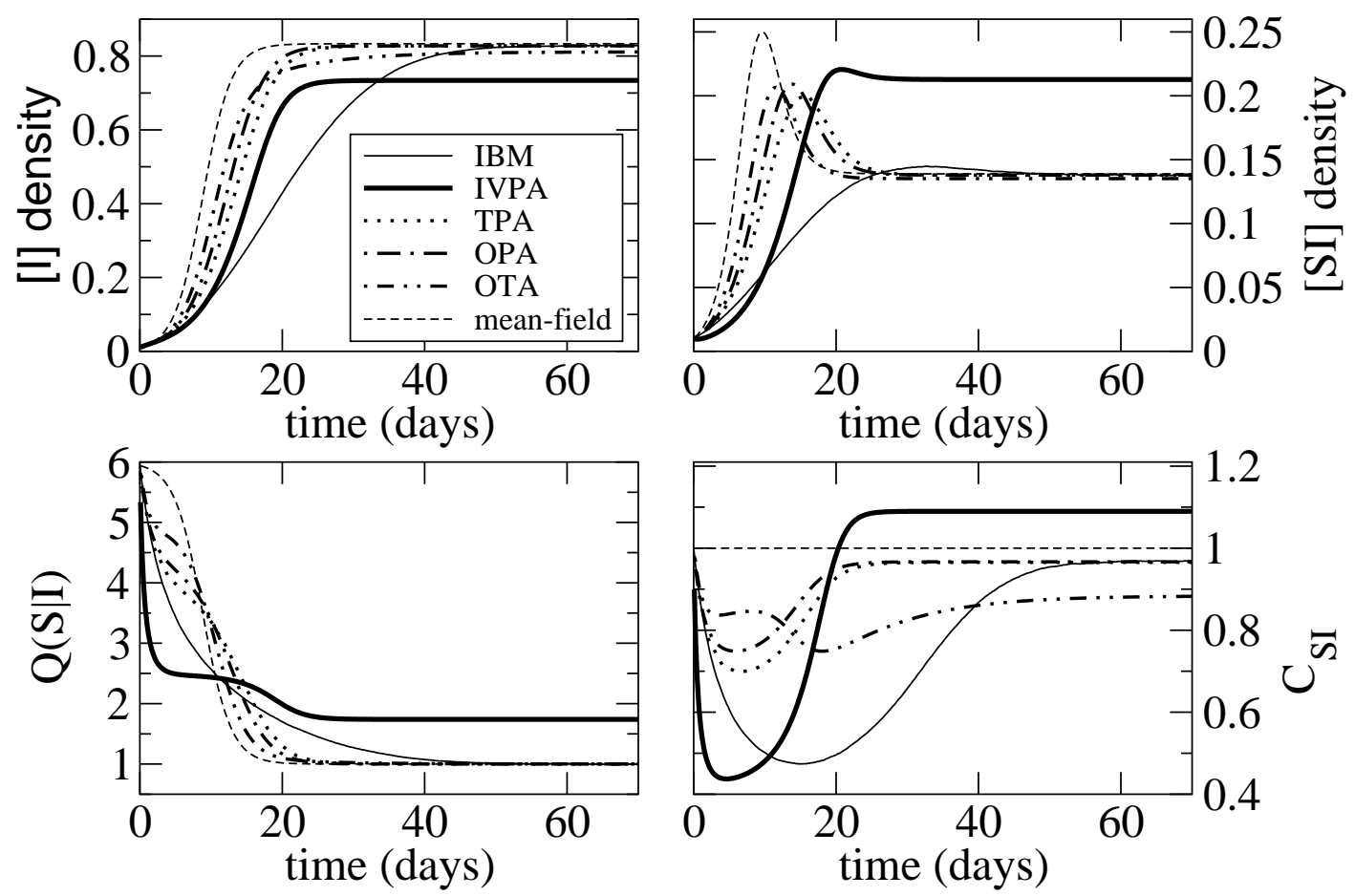


\section{Appendix A: Derivation of pairwise equations of motion}

To derive the equation of motion for some quantity $g$, say, we use the following master equation which relates the state $\sigma$ of the lattice at time $t$ to the rate of change in $g$ :

$$
\frac{d}{d t} g(\sigma)=\sum_{e \in \text { events }} r(e) \Delta g_{e}(\sigma) .
$$

where the sum is over all events $e$ in the lattice, and where each event occurs at rate $r(e)$ and changes $g(\sigma)$ by an amount $\Delta g_{e}(\sigma)$.

Here we derive the equation of motion for $[S I]$, the number of susceptible-infected pairs. First we list all events which will impact $[S I]$ :

$$
\begin{array}{ll}
S I \rightarrow S S & \text { recovery } \\
S I \rightarrow I I & \text { infection } \\
S S \rightarrow S I & \text { infection } \\
I I \rightarrow S I & \text { recovery }
\end{array}
$$

Using Eq. (55) and the above list we obtain:

$$
\begin{aligned}
\frac{d}{d t}[S I]= & \sum_{\sigma_{x}=S}(\nu)\left(-Q_{x}(I)\right)+\sum_{\sigma_{x}=I}(\nu)\left(+Q_{x}(I)\right) \\
& +\sum_{\sigma_{x y}=S I}\left(\lambda Q_{x y}(I)\right)(-1)+\sum_{\sigma_{x y}=S S}\left(\lambda Q_{x y}(I)\right)(+1)
\end{aligned}
$$

where $Q_{x}(I)$ is the number of infected neighbors of site $x, Q_{x y}(I)$ is the number of infected neighbors of pair $x y$, and $\sigma_{x}$ (resp. $\sigma_{x y}$ ) denotes the state of site $x$ (resp. pair $x y)$.

Next we make the following substitutions for $Q_{x}(I)$ and $Q_{x y}(I)$ :

$$
\begin{array}{rlrl}
Q_{x}(I) & =Q(I \mid S)+\eta_{x}(I \mid S) & \sigma_{x}=S \\
Q_{x y}(I)=Q(I \mid S I)+\eta_{x y}(I \mid S I) & \sigma_{x y}=S I .
\end{array}
$$

where $Q(I \mid S)$ (resp. $Q(I \mid S I)$ ) is the population-averaged mean of the number of infected neighbors of a susceptible (resp. of a susceptible-infected pair) and $\eta_{x}(I \mid S)$ 
(resp. $\eta_{x y}(I \mid S I)$ ) is the deviation from the population-averaged value of $Q_{x}(I)$ at $x$ (resp. $Q_{x y}(I)$ at pair $x y$ ). Substituting Eqs. (57) and (58) into Eq. (55) gives

$$
\begin{aligned}
\frac{d}{d t}[S I]= & -\sum_{\sigma_{x}=S} \nu\left(Q(I \mid S)+\eta_{x}(I \mid S)\right)+\sum_{\sigma_{x}=I} \nu\left(Q(I \mid I)+\eta_{x}(I \mid I)\right) \\
& -\sum_{\sigma_{x y}=S I} \lambda\left(Q(I \mid S I)+\eta_{x y}(I \mid S I)\right) \\
& +\sum_{\sigma_{x y}=S S} \lambda\left(Q(I \mid S S)+\eta_{x y}(I \mid S S)\right) .
\end{aligned}
$$

The population-averaged quantities $Q(I \mid S), Q(I \mid I), Q(I \mid S I)$ and $Q(I \mid S S)$

are constant and hence can be taken out of the sums. Also the terms such as $\sum_{\sigma_{x}=S} \eta_{x}(I \mid S)$ which represent fluctuations sum to zero by definition. Evaluating the sums accordingly produces

$$
\frac{d}{d t}[S I]=-\nu[S I]+\nu[I I]-\lambda[S I] Q(I \mid S I)+\lambda[S S] Q(I \mid S S) .
$$

The third-order terms $Q(I \mid S I)$ and $Q(I \mid S S)$ must be expressed in terms of pair variables; see Sections 2 and 3 of the main text for a discussion of this closure procedure.

\section{Appendix B: Derivation of $\mathcal{R}_{0}$ under the Invasory pair approximation}

As before, the equation of motion for $C_{S I}$ is

$$
\frac{d}{d t} C_{S I}=\frac{N}{Q}\left[\frac{1}{[S][I]} \frac{d}{d t}[S I]+\frac{[S I]}{[S][I]}\left(-\frac{1}{[I]} \frac{d}{d t}[I]-\frac{1}{[S]} \frac{d}{d t}[S]\right)\right]
$$

Substituting the IVPA equations of motion

$$
\begin{aligned}
\frac{d}{d t}[S I] & =-\nu[S I]+\nu[I I]-\lambda[S I]\left(1+\frac{Q-1}{Q} \frac{\lambda}{\nu} \frac{[S I]}{[I]}\right)+\lambda \frac{Q-1}{Q} \frac{[S S][S I]}{[S]} \\
\frac{d}{d t}[I I] & =-2 \nu[I I]+2 \lambda[S I]\left(1+\frac{Q-1}{Q} \frac{\lambda}{\nu} \frac{[S I]}{[I]}\right) \\
\frac{d}{d t}[I] & =-\nu[I]+\lambda[S I]
\end{aligned}
$$

and then re-expressing the equations in terms of $C_{S I}$ and $C_{I I}$ produces

$$
\begin{aligned}
\frac{d}{d t} C_{S I}= & \nu \frac{N}{[S]}\left(\frac{[I] C_{I I}}{N}\right)-\lambda C_{S I}-\frac{\lambda^{2}}{\nu} \frac{[S]}{N}(Q-1) C_{S I}^{2}+\lambda(Q-1) \frac{[S]}{N} C_{S S} C_{S I} \\
& -\lambda Q \frac{[S]}{N} C_{S I}^{2}-\nu \frac{[I]}{[S]} C_{S I} .
\end{aligned}
$$


In the early stages of invasion we have $[S] \approx N$ and $[I] / N,[S I] / N \ll 1$, hence Eq. (63) becomes

$$
\frac{d}{d t} C_{S I}=\nu\left(\frac{[I] C_{I I}}{N}\right)-\lambda C_{S I}-\frac{\lambda^{2}}{\nu}(Q-1) C_{S I}^{2}+\lambda(Q-1) C_{S I}-\lambda Q C_{S I}^{2} .
$$

The quantity $\left([I] C_{I I} / N\right)$ is of order one, and so in a similar fashion we derive the equation of motion for $\left([I] C_{I I} / N\right)$ :

$$
\begin{aligned}
\frac{d}{d t}\left(\frac{[I] C_{I I}}{N}\right)= & \frac{1}{Q}\left(\frac{1}{[I]} \frac{d}{d t}[I I]-\frac{[I I]}{[I]^{2}} \frac{d}{d t}[I]\right) \\
= & -\nu\left(\frac{[I] C_{I I}}{N}\right)+2 \lambda C_{S I} \frac{[S]}{N}+2 \frac{\lambda^{2}}{\nu} \frac{[S]^{2}}{N^{2}}(Q-1) C_{S I}^{2} \\
& -\lambda Q \frac{[S]}{N} C_{S I}\left(\frac{[I] C_{I I}}{N}\right)
\end{aligned}
$$

As before, during the early stages of invasion, $[S] \approx N$ and $[I] / N \ll 1$ and so this becomes

$$
\frac{d}{d t}\left(\frac{[I] C_{I I}}{N}\right)=-\nu\left(\frac{[I] C_{I I}}{N}\right)+2 \lambda C_{S I}+2 \frac{\lambda^{2}}{\nu} C_{S I}^{2}(Q-1)-\lambda Q C_{S I}\left(\frac{[I] C_{I I}}{N}\right) .
$$

Setting the left hand sides of Eqs. (46) and (48) equal to zero and solving for $C_{S I}$ and $\left([I] C_{I I} / N\right)$ yields the pseudo-equilibrium

$$
\begin{aligned}
C_{S I}^{p e q}= & \frac{\nu}{\lambda Q} \frac{\sqrt{\lambda^{2}\left(Q^{4}+2 Q^{3}-5 Q^{2}+2 Q+1\right)+2 \lambda \nu Q\left(Q^{2}+Q+1\right)+Q^{2} \nu^{2}}}{2(\lambda Q-\lambda+\nu Q)} \\
& +\frac{\nu}{\lambda Q} \frac{-\lambda Q-\lambda-\nu Q+\lambda Q^{2}}{2(\lambda Q-\lambda+\nu Q)} \\
\left(\frac{[I] C_{I I}}{N}\right)^{p e q}= & 1-C_{S I}^{p e q} .
\end{aligned}
$$

And using $\mathcal{R}_{0}=\beta C_{S I} / \nu$ we obtain the expression for $\mathcal{R}_{0}$ under the IVPA (Eq. (51)). 